\title{
Hot pixel contamination in the CMB correlation function?
}

\author{
R. Aurich, S. Lustig, and F. Steiner \\ Institut für Theoretische Physik, Universität Ulm, \\ Albert-Einstein-Allee 11, D-89069 Ulm, Germany
}

\begin{abstract}
Recently, it was suggested that the map-making procedure, which is applied to the time-ordered CMB data by the WMAP team, might be flawed by hot pixels. This could lead to a bias in the pixels having an angular distance of about $141^{\circ}$ from hot pixels due to the differential measuring process of the satellite WMAP. Here, the bias is confirmed, and the temperature two-point correlation function $C(\vartheta)$ is reevaluated by excluding the affected pixels. It is shown that the most significant effect occurs in $C(\vartheta)$ at the largest angles near $\vartheta=180^{\circ}$. Furthermore, the corrected correlation function $C(\vartheta)$ is applied to the cubic topology of the Universe, and it is found that such a multi-connected universe matches the temperature correlation better than the $\Lambda \mathrm{CDM}$ concordance model, provided the cubic length scale is close to $L=4$ measured in units of the Hubble length.

PACS numbers: 98.80.-k, 98.70.Vc, 98.80.Es
\end{abstract}

\section{Introduction}

One of the most important observational inputs of modern cosmology is provided by the cosmic microwave background (CMB). The sky maps produced by the WMAP team [1] have found wide applications in cosmological parameter extraction and provided several surprises. Besides the low power at large scales, which had already been discovered by COBE [2], some strange properties emerged concerning the statistical isotropy, i.e. an anomalous alignment between the quadrupole and the octopole [3, 4] as well as an asymmetry of the CMB fluctuations within the two hemispheres with respect to the ecliptic plane [5]. Both anomalies are difficult to reconcile within the standard inflationary framework. A possible explanation could be related to the uncertainties which inevitably arise in the foreground removal procedures [6].

Recently, Liu and Li [7, 8] have discussed a possible systematics which lies at the heart of the map-making procedure. The main point arises from the fact that WMAP measures temperature differences at two points on the sphere separated by about $141^{\circ}$ which is due to the construction of the probe. They demonstrate in [7, 8] that the pixels, which lie on a scan ring having diameter $141^{\circ}$ with a hot pixel at its centre, systematically obtain a lower temperature. They show that the corresponding crosscorrelation function displays a negative correlation at the crucial angle of $141^{\circ}$ in the 


\begin{tabular}{|c|c|c|c|c|}
\hline channel & $\mathrm{Q} 1, \mathrm{Q} 2$ & $\mathrm{~V} 1, \mathrm{~V} 2$ & $\mathrm{~W} 1, \mathrm{~W} 4$ & $\mathrm{~W} 2, \mathrm{~W} 3$ \\
\hline diameter & $144.3^{\circ}$ & $140.5^{\circ}$ & $139.8^{\circ}$ & $142.0^{\circ}$ \\
\hline
\end{tabular}

Table 1. The diameters of the scan rings in dependece on the channels 9 .

three frequency bands $\mathrm{Q}, \mathrm{V}$, and $\mathrm{W}$ thus pointing to a systematic distortion occurring in all three bands. In order to estimate the magnitude of the induced temperature distortion, they [7, 8] select the 2000 hottest pixels for a detailed analysis and find a temperature distortion in the range between $11 \mu \mathrm{K}$ and $14 \mu \mathrm{K}$ which in turn translates into a $10 \%$ difference in the quadrupole and the octopole moments. This systematics is not remedied by a prolonged observation time. It should be noted, however, that the WMAP team [1] computes the average temperature of pixels, which do not belong to the set of hot pixels, by omitting the hot pixels using the processing mask, so that it is not clear at which point of the map-making pipeline this systematics arises.

In this paper, we construct a modified mask in order to check whether the suggested systematics really exists. Furthermore, the two-point temperature correlation function $C(\vartheta)$ is investigated with respect to this systematics. With the temperature correlation function, which is based only on those pixels that are not strongly affected by this systematics, we carry out a test of a cubic topology of our Universe.

\section{A modified Liu-Li mask}

The hot pixel systematics does not affect all pixels on the corresponding scan rings in the same way. To each hot pixel belongs a well defined scan ring, of course, but due to the large number of hot pixels there is an equally large number of affected scan rings, which intersect each other, in general. This is due to the large size of the scan rings having diameters between $135^{\circ}$ and $144^{\circ}$ depending on the channel [9], see Table 1 for the individual values. Since the suggested systematics leads to a negative bias on a given scan ring, the bias should be more pronounced at the intersections of several affected scan rings. In order to test this effect, a modified Liu-Li mask is constructed by the following procedure.

We start with the five-year Q1, Q2, V1, V2, W1, W2, W3, W4 channel sky maps which are not foreground reduced and thus contain the hot pixels. These maps as well as the KQ75 and KQ85 masks are provided by the WMAP team [1] on the LAMBDA home page lambda.gsfc.nasa.gov. Then all pixels above a given temperature threshold $T_{\text {thres }}$ are selected as hot pixels and their corresponding scan rings with the diameters given in Table 1 are computed. In this way 8 maps are obtained whose pixel values contain the number $N_{\text {inter }}$ of corresponding hot pixels. From these 8 maps we compute 3 average maps, one for each of the three bands $\mathrm{Q}, \mathrm{V}$, and $\mathrm{W}$ with a mean $N_{\text {inter }}$. If a given pixel obtains more than $N_{\text {inter }}$ intersections from affected scan rings, it gets masked out. This mask depends then on both thresholds $T_{\text {thres }}$ and $N_{\text {inter }}$. In a last step all pixels within the KQ75 or KQ85 mask are rejected. The remaining pixels should be those being less 


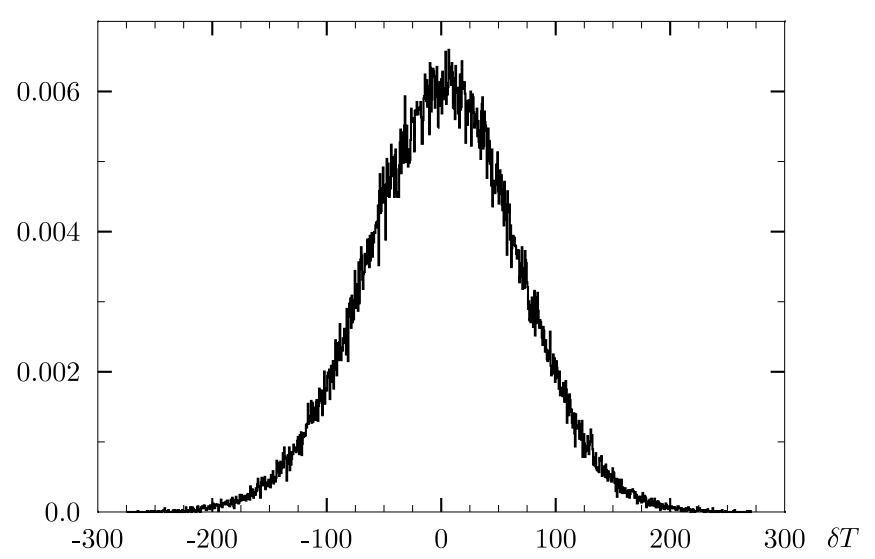

Figure 1. The distribution of the CMB temperature values $\delta T$ in $\mu \mathrm{K}$ is shown for the ILC (5yr) map by taking into account only the pixels outside the KQ75 mask. The temperature distribution has a standard deviation of $66 \mu \mathrm{K}$.

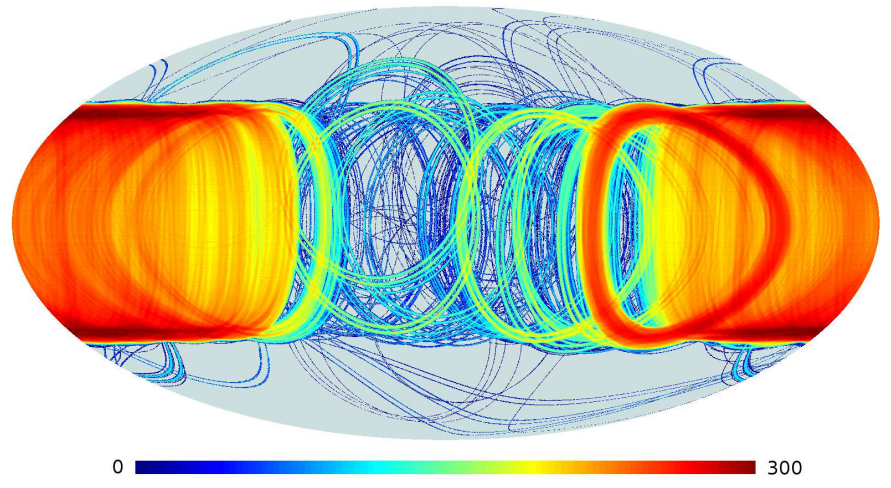

Figure 2. The degree of the disturbance by hot pixels is shown for the threshold $T_{\text {thres }}=1000 \mu \mathrm{K}$ in Galactic coordinates. The pixels are represented in dependence on the number $N_{\text {inter }}$ of scan rings with respect to the $\mathrm{W}$ band belonging to hot pixels with $\delta T>T_{\text {thres }}$ up to $N_{\text {inter }}=300$ (colour online).

affected by foregrounds and therefore containing the safest cosmological information.

The value of the temperature threshold $T_{\text {thres }}$ is chosen to be many times larger than the typical temperature fluctuation of the cosmological signal. In Figure 1 the distribution of the temperature values $\delta T$ is shown for the ILC map based on the fiveyear data [1] by taking into account only the pixels outside the KQ75 mask. The temperature distribution has a standard deviation of $66 \mu \mathrm{K}$. We choose in the following three thresholds of $1000 \mu \mathrm{K}, 2000 \mu \mathrm{K}$, and $4000 \mu \mathrm{K}$, respectively, which are very large compared to the standard deviation of $66 \mu \mathrm{K}$ of the $\mathrm{CMB}$ signal. In Figure 2 we show the distribution of the affected pixels for the $\mathrm{W}$ band for a hot pixel threshold of $T_{\text {thres }}=1000 \mu \mathrm{K}$, where the Mollweide projection is used in Galactic coordinates. The intensity of the pixels depends on the number $N_{\text {inter }}$ of hits by scan rings of hot pixels. The scale is truncated at $N_{\text {inter }}=300$. One observes that the most critical pixels lie below and above the Galactic plane opposite to the Galactic centre which is in the centre of Figure 2. This behaviour is caused by the large diameter of $\sim 141^{\circ}$ of the scan 


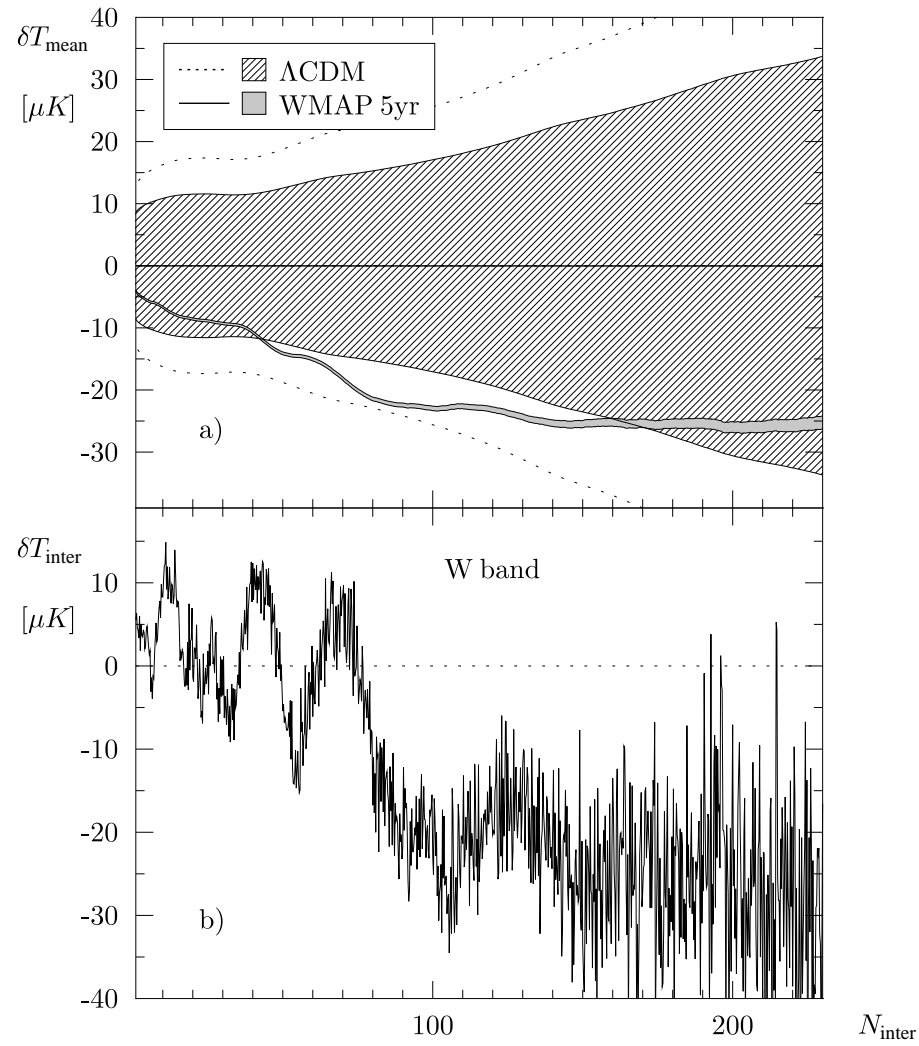

Figure 3. The mean temperature $\delta T_{\text {mean }}$ is shown for the $\mathrm{W}$ band foreground-reduced map (5yr). The mean value is computed from the pixels inside the modified Liu-Li mask for the $\mathrm{W}$ band with $T_{\text {thres }}=1000 \mu \mathrm{K}$ but outside the KQ75 mask in dependence on the threshold $N_{\text {inter. }}$. In Panel a) the value of $\delta T_{\text {mean }}$ is computed from those pixels which are related to at least $N_{\text {inter }}$ hot pixels. The grey band represents the $2 \sigma$ uncertainty of the detector noise in the corresponding area. The shaded region denotes the $2 \sigma$ uncertainty obtained from 100000 simulations of $\Lambda$ CDM models subjected to the same procedure. The dotted curves show the $3 \sigma$ uncertainties. In Panel b) the mean temperature $\delta T_{\text {inter }}$ is computed from pixels corresponding to exactly $N_{\text {inter }}$ hot pixels.

rings and the fact that many hot pixels above $T_{\text {thres }}$ are near the Galactic centre. The modulus of Galactic latitude of the domain with the largest values of $N_{\text {inter }}$ is given by $180^{\circ}-141^{\circ}=39^{\circ}$ revealed by the dark regions in Figure 2 ,

In order to verify the negative-temperature bias of the pixels belonging to hot pixel scan rings, the mean temperature $\delta T_{\text {mean }}$ is computed inside the modified Liu-Li mask with $T_{\text {thres }}=1000 \mu \mathrm{K}$ but outside the KQ75 mask. The mean $\delta T_{\text {mean }}$ depends on the chosen value of the threshold number $N_{\text {inter }}$, i. e. pixels are selected if they are related to at least $N_{\text {inter }}$ hot pixels. This procedure tests whether the negative bias increases with the number $N_{\text {inter }}$ of scan rings. Figure 3 a reveals that $\delta T_{\text {mean }}$ computed for the W band foreground-reduced map indeed drops towards more negative values until a value of $N_{\text {inter }} \simeq 140$ is reached. The pixels with $N_{\text {inter }} \gtrsim 140$ possess a temperature bias of the order of $-25 \mu \mathrm{K}$. This is the expected behaviour since with increasing $N_{\text {inter }}$ only the most severely affected pixels are taken into account, i. e. those with the largest 


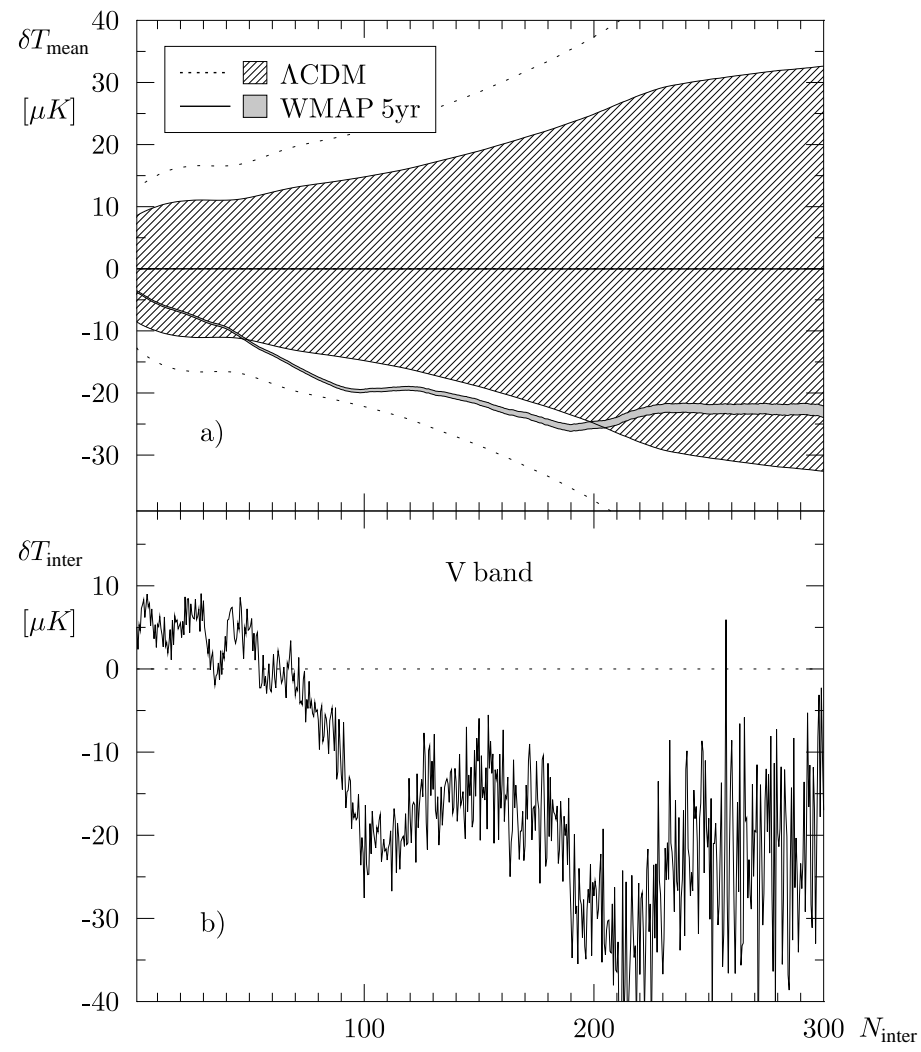

Figure 4. As in Figure 3 the mean temperatures $\delta T_{\text {mean }}$ and $\delta T_{\text {inter }}$ are shown but for the $\mathrm{V}$ band foreground-reduced map (5yr).

systematics. Whereas panel a) displays $\delta T_{\text {mean }}$ computed from those pixels having at least $N_{\text {inter }}$ hot pixels, panel b) shows the mean value $\delta T_{\text {inter }}$ computed only from those pixels having exactly $N_{\text {inter }}$ hot pixels. The number of the latter ones is lower and thus the curve possesses larger statistical fluctuations. But it nevertheless demonstrates the negative bias for pixels with $N_{\text {inter }}$ above 100 .

In order to check the significance of this result, 100000 simulations of $\Lambda \mathrm{CDM}$ concordance models are generated for which $\delta T_{\text {mean }}$ is computed using the same procedure as outlined above. These values scatter symmetrically around a zero mean. The shaded region in Figure 3 a denotes the $2 \sigma$ deviation from the zero mean. One observes that the $\mathrm{W}$ band distortion is over a wide range of values of $N_{\text {inter }}$ larger than the $2 \sigma$ deviation for $\Lambda \mathrm{CDM}$ models. This demonstrates that there is a systematics for these pixels.

Figures 4 and 5 present the result of the same analysis as shown in Figure 3, but for the $\mathrm{V}$ and $\mathrm{Q}$ bands, respectively. In both frequency bands the same negative bias of at least $2 \sigma$ is observed when $N_{\text {inter }}$ is sufficiently large, i. e. when enough hot pixels are connected to the considered one. For very large values of $N_{\text {inter }}$ the deviation is smaller than $2 \sigma$ but very few pixels occur in that range. The values of $N_{\text {inter }}$ cannot be directly compared between the different channels since they possess different hot pixels lying above $T_{\text {thres }}$ because of the different foregrounds. In the case of the $\mathrm{W}$ band 


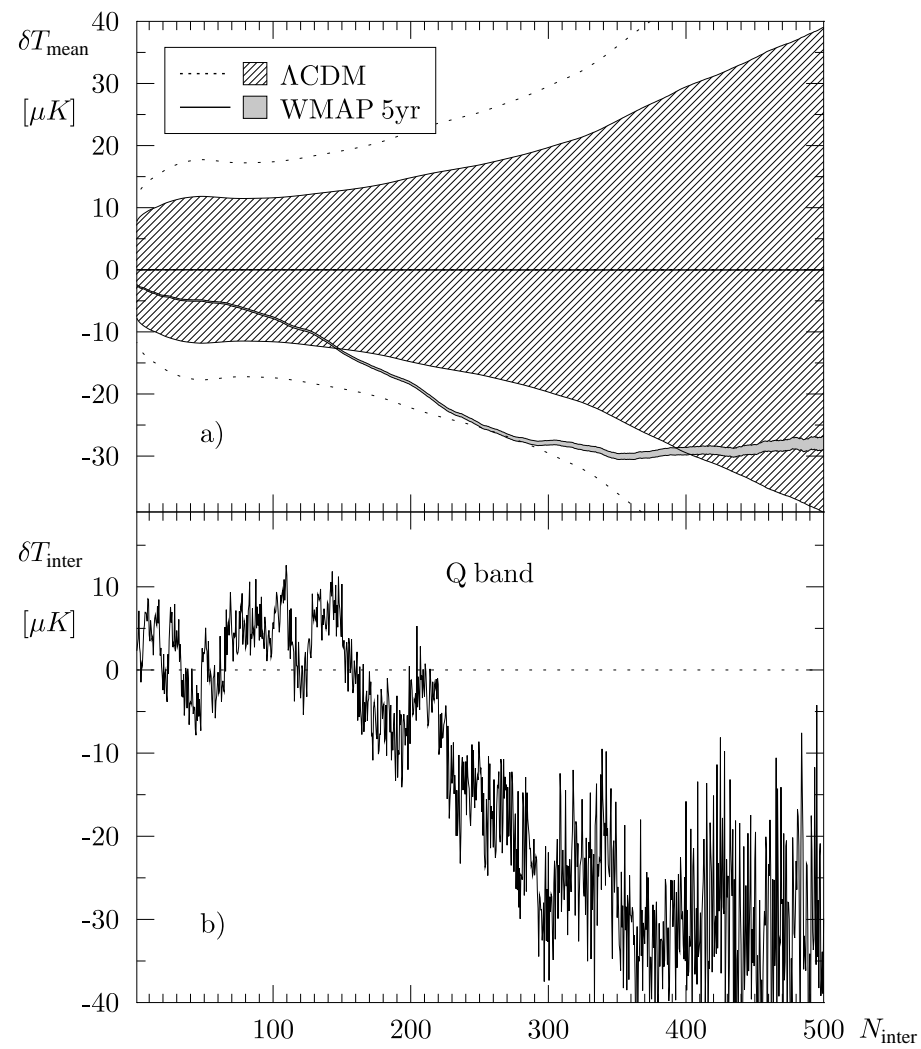

Figure 5. As in Figure 3 the mean temperatures $\delta T_{\text {mean }}$ and $\delta T_{\text {inter }}$ are shown but for the $\mathrm{Q}$ band foreground-reduced map (5yr).

the dust contribution dominates whereas the synchrotron and free-free emission is more important in the case of the $\mathrm{V}$ and $\mathrm{Q}$ bands.

None of our 100000 simulations of $\Lambda$ CDM concordance models yields a $\delta T_{\text {mean }}$ curve which always has values below the WMAP result shown in the panel a) of Figures 3 5. The same result is obtained for $\delta T_{\text {inter }}$ shown in the corresponding panel b). This emphasises the peculiarity of the negative-temperature bias.

\section{The correlation hole at $\vartheta=180^{\circ}$}

Let us now come to the temperature two-point correlation function $C(\vartheta)$, which is defined as

$$
C(\vartheta):=\left\langle\delta T(\hat{n}) \delta T\left(\hat{n}^{\prime}\right)\right\rangle \quad \text { with } \quad \hat{n} \cdot \hat{n}^{\prime}=\cos \vartheta
$$

It was computed by 2 from the COBE 4yr data and was found to display unexpectedly low power at large angles $\vartheta \gtrsim 60^{\circ}$. At these large angles $C(\vartheta)$ is close to zero. Only near $\vartheta=180^{\circ}$ it shows a slight negative power, which is much more pronounced in the WMAP five-year data [10]. This anticorrelation of antipodal points is the so-called "correlation hole". Copi et al. [10] show the strong dependence of the correlation hole on the chosen mask. The correlation function $C(\vartheta)$ possesses the largest antipodal anticorrelation if one uses no mask at all, whereas applying the KQ75 mask reduces 


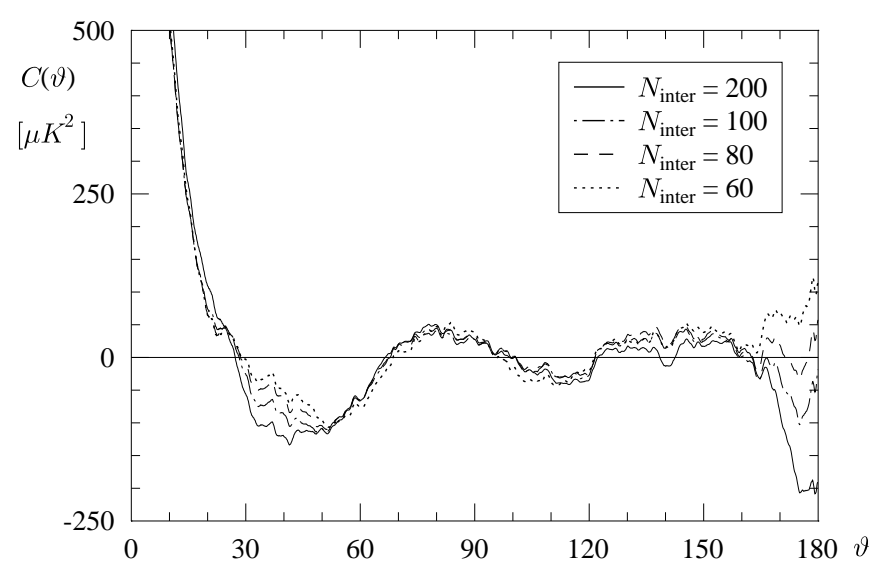

Figure 6. The correlation function $C(\vartheta)$ is computed from the $\mathrm{W}$ band foregroundreduced map (5yr) using the pixels outside the KQ75 mask. In addition the pixels inside the modified Liu-Li mask are rejected for 4 different values of the threshold $N_{\text {inter }}$.

the anticorrelation, but it is nevertheless present. They conclude that the full-sky results seem inconsistent with the cut-sky results. Furthermore, most of the large-angle correlation in reconstructed sky maps lies inside the part of the sky that is contaminated by the Galaxy.

We do not take into account the cosmic variance which arises on the theoretical side since the cosmic initial conditions are assumed only on statistical grounds. Here, we are interested in the correlation function $C(\vartheta)$ observed from our special observer point within our Universe.

We now show that the remaining negative correlation near $\vartheta=180^{\circ}$ is caused by the pixels lying within the modified Liu-Li mask. To that aim we apply our modified Liu-Li mask with $T_{\text {thres }}=1000 \mu \mathrm{K}$ together with the KQ75 mask to the $\mathrm{W}$ band foregroundreduced map (5yr) for several choices of the intersection threshold $N_{\text {inter }}$. The result shown in Figure 6 reveals a pronounced correlation hole for large values of $N_{\text {inter }}$, i. e. for those cases where only a small number of further pixels is rejected after applying the KQ75 mask. The curve belonging to $N_{\text {inter }}=200$ displays almost the usual result obtained by using only the KQ75 mask, whereas the one belonging to $N_{\text {inter }}=60$ shows even positive correlations near $\vartheta=180^{\circ}$. The four cases presented in Figure [6] also demonstrate that the correlation is at other angular separations $\vartheta$ nearly independent on the value $N_{\text {inter }}$ except for the interval $\left[30^{\circ}, 50^{\circ}\right]$. However, the strongest sensitivity occurs at $\vartheta=180^{\circ}$.

Let us now address the question how probable such a behaviour is with respect to the $\Lambda$ CDM concordance model. In order to compute the probability that $C(\vartheta)$ remains nearly unchanged for $50^{\circ}<\vartheta<165^{\circ}$, define the difference

$$
\Delta C(\vartheta):=C_{\mathrm{KQ} 75+\mathrm{LL}}(\vartheta)-C_{\mathrm{KQ} 75}(\vartheta)
$$

between $C(\vartheta)$ using the KQ75 mask and the modified Liu-Li mask for $N_{\text {inter }}=60$, which is denoted as $C_{\mathrm{KQ75+LL}}(\vartheta)$, and $C(\vartheta)$ using only the KQ75 mask denoted as $C_{\mathrm{KQ75}}(\vartheta)$. 


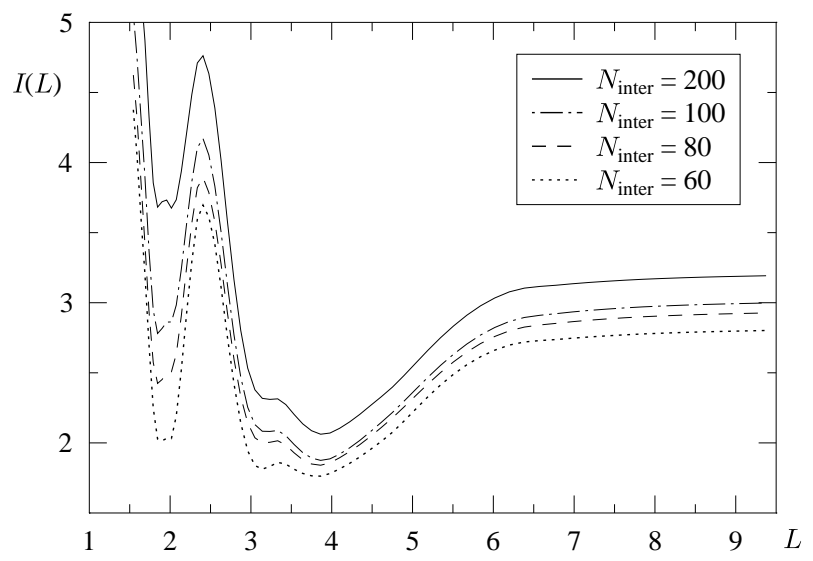

Figure 7. The weighted temperature correlation difference $I(L)$ is computed from the four correlation functions $C(\vartheta)$ shown in Figure 6. The length $L$ denotes the size of the cubic topology measured in units of the Hubble length.

The integrated quantity

$$
D:=\int_{\vartheta=50^{\circ}}^{\vartheta=165^{\circ}} d \cos \vartheta|\Delta C(\vartheta)|
$$

measures how strongly $C(\vartheta)$ is affected by the suspected pixels. The value obtained from the $\mathrm{W}$ band data is surprisingly low, indeed from our $100000 \Lambda \mathrm{CDM}$ concordance models only 495 models possess a smaller value, i. e. $D_{\Lambda \mathrm{CDM}}<D_{\mathrm{WMAP}}$. Thus, $99.5 \%$ of the $\Lambda \mathrm{CDM}$ models show a stronger change in $C(\vartheta)$ than the considered WMAP data. Concerning the correlation hole, only 3111 models satisfy $\Delta C_{\Lambda \mathrm{CDM}}\left(180^{\circ}\right)>$ $\Delta C_{\mathrm{WMAP}}\left(180^{\circ}\right)$, i. e. the large increase of $C(\vartheta)$ at $\vartheta=180^{\circ}$ is also unusual at the 3 percent level. If one requires both conditions simultaneously not a single $\Lambda$ CDM model remains. This emphasises the unusual behaviour of the suspected pixels.

\section{The cubic topology for the modified correlation function}

The surprisingly low CMB large-angle power of the correlation function $C(\vartheta)$, which was discovered in [2], is at variance with the $\Lambda \mathrm{CDM}$ concordance model as has been found in [11 and recently emphasised in [12] and [10]. One of the possible explanations for this unexpected behaviour is provided by models of the Universe whose spatial section is represented by a multi-connected space form. For a general introduction to the topology with respect to cosmology, see e.g. [13, 14, 15, 16, 17, 18. In these models exists naturally a largest wavelength at which perturbations can occur as seeds for structure formation. This wavelength is mainly determined by the size of the topological space form. The correlation function $C(\vartheta)$ has been studied for several multi-connected space forms, and a low large-angle power has been shown to exist in the case of the hyperbolic Picard topology [19, 20], the spherical Poincaré dodecahedron [21], and the flat toroidal universe [12].

In [12] we have analysed the WMAP three-year data with respect to the question 
whether a flat toroidal universe, i. e. a cubic topology, is compatible with the CMB observations. To that aim the correlation function $C^{\text {model }}(\vartheta)$ obtained from the cubic topology with side length $L$ is compared to the observed correlation function $C^{\text {obs }}(\vartheta)$ by using the integrated weighted temperature correlation difference

$$
I:=\int_{-1}^{1} d \cos \vartheta \frac{\left(C^{\text {model }}(\vartheta)-C^{\text {obs }}(\vartheta)\right)^{2}}{\operatorname{Var}\left(C^{\operatorname{model}}(\vartheta)\right)} .
$$

It is found in 12 that the results depend on the chosen mask that is applied to the CMB data. Applying the kp0 mask to the data shows that cubic topologies around $L=3.86$ are preferred whereas using no mask at all leads to models having a size around $L=4.35$. ( $L$ is given in units of the Hubble length $L_{\mathrm{H}}=c / H_{0} \simeq 4.26 \mathrm{Gpc}$ for $h=0.704$.) This demonstrates the sensitivity on the pixels that are selected for the computation of the correlation function $C(\vartheta)$.

Instead of using the three-year data as in [12], we now compute the measure $I(L)$, eq. (4), using the updated five-year data, where we use the $\mathrm{W}$ band foreground reduced map. Furthermore, we apply the KQ75 mask together with the four modified Liu-Li masks discussed above. The result shown in Figure 7 is that cubic topologies slightly smaller than $L=4$ lead to the smallest values of $I(L)$, i. e. lead to the best agreement with the data. The corresponding values for the $\Lambda \mathrm{CDM}$ concordance model having infinite volume can also be read off from Figure 7. Since the distance to the surface of last scattering (SLS) is $L_{\mathrm{SLS}}=\Delta \eta L_{\mathrm{H}} \simeq 14.2 \mathrm{Gpc}$ where $\Delta \eta=\eta_{0}-\eta_{\mathrm{SLS}}=3.329(\eta$ is the conformal time), topologies above $L \simeq 6.6$ are so large that the SLS fits completely

inside the toroidal universe. For larger models the value of $I(L)$ saturates at the infinite limit $L \rightarrow \infty$ corresponding to the concordance model. For all four values of $N_{\text {inter }}$ the correlation function $C(\vartheta)$ is in much better agreement with a cubic universe with $L$ slightly below 4 than with the infinite $\Lambda$ CDM concordance model. The application of the modified Liu-Li mask has little influence on the ratio of $I(L) / I(\Lambda \mathrm{CDM})$ for the minimum near $L=4$ which is due to the fact that $C(\vartheta)$ is practically unchanged over the large interval from $\vartheta \simeq 50^{\circ}$ up to $\vartheta \simeq 165^{\circ}$ and below $\vartheta \simeq 30^{\circ}$. Excluding more pixels, i. e. decreasing $N_{\text {inter }}$ form 200 to 60 , produces a more pronounced second minimum around $L \simeq 2$ which lies for $N_{\text {inter }}=60$ below the value of the concordance model but above the absolute minimum near $L=4$. This new feature is caused by the diminished power of $C(\vartheta)$ for angles $\vartheta$ in the interval $\left[30^{\circ}, 50^{\circ}\right]$ leading to a $C(\vartheta)$ with a power suppression already above $\vartheta \simeq 30^{\circ}$ instead of the usual $60^{\circ}$.

\section{Summary}

The presently best full-sky maps of the cosmic microwave background radiation are obtained by WMAP whose receivers are differential radiometers measuring the difference between two telescope beams separated by about $141^{\circ}$. Liu and Li [7, 8] discuss the possibility that the inclusion of hot pixels in the map-making process causes a bias in those pixels corresponding to a scan ring of about $141^{\circ}$. Due to the large diameter of the scan rings, the affected pixels lie in a region far from the galactic plane which is usually 
considered to be scarcely contaminated. Here we confirm the bias found in [7, 8] and, furthermore, compute the temperature two-point correlation function $C(\vartheta)$ by taking this bias into account. The most dramatic effect of these biased pixels is the so-called correlation hole at $\vartheta=180^{\circ}$ in $C(\vartheta)$, which is absent by eliminating sufficiently many affected pixels. Thus, a correlation function $C(\vartheta)$ is obtained which displays almost no correlations above all angular scales larger than $60^{\circ}$.

In Figures 17, we have presented our results for the modified Liu-Li mask using the threshold temperature $T_{\text {thres }}=1000 \mu \mathrm{K}$ and employing the KQ75 mask. We have checked that the conclusions of this paper remain the same if the KQ85 mask is used instead of the KQ75 mask and, furthermore, if the calculations are performed for the thresholds $2000 \mu \mathrm{K}$ and $4000 \mu \mathrm{K}$, respectively.

Using the correlation function $C(\vartheta)$ based on the most reliable data, a test for a universe with a cubic topology is carried out. It is found that such a model fits $C(\vartheta)$ better than the $\Lambda \mathrm{CDM}$ concordance model if the side length $L$ of the cubic fundamental cell is slightly smaller than $L=4$ measured in units of the Hubble length.

\section{Acknowledgments}

HEALPix (healpix.jpl.nasa.gov) 22] and the WMAP data from the LAMBDA website (lambda.gsfc.nasa.gov) were used in this work. The computations are carried out on the Baden-Württemberg grid (bwGRiD).

\section{References}

[1] G. Hinshaw et al., Astrophys. J. Supp. 180, 225 (2009), arXiv:0803.0732 [astro-ph].

[2] G. Hinshaw et al., Astrophys. J. Lett. 464, L25 (1996).

[3] M. Tegmark, A. de Oliveira-Costa, and A. J. S. Hamilton, Phys. Rev. D 68, 123523 (2003), astro-ph/0302496.

[4] A. de Oliveira-Costa, M. Tegmark, M. Zaldarriaga, and A. Hamilton, Phys. Rev. D 69, 063516 (2004), astro-ph/0307282.

[5] H. K. Eriksen, F. K. Hansen, A. J. Banday, K. M. Górski, and P. B. Lilje, Astrophys. J. 605, 14 (2004).

[6] B. Gold et al., Astrophys. J. Supp. 180, 265 (2009), arXiv:0803.0715 [astro-ph].

[7] H. Liu and T.-P. Li, arXiv:0806.4493 [astro-ph] (2008).

[8] H. Liu and T.-P. Li, Sci. China G-Phy. Mech. Astron. 52, 804 (2009), arXiv:0809.4160 [astro-ph].

[9] G. Hinshaw et al., Astrophys. J. Supp. 148, 63 (2003), arXiv:astro-ph/0302222.

[10] C. J. Copi, D. Huterer, D. J. Schwarz, and G. D. Starkman, Mon. Not. R. Astron. Soc. 399, 295 (2009), arXiv:0808.3767 [astro-ph].

[11] D. N. Spergel et al., Astrophys. J. Supp. 148, 175 (2003), astro-ph/0302209.

[12] R. Aurich, H. S. Janzer, S. Lustig, and F. Steiner, Class. Quantum Grav. 25, 125006 (2008), arXiv:0708.1420 [astro-ph].

[13] M. Lachièze-Rey and J. Luminet, Physics Report 254, 135 (1995).

[14] G. D. Starkman, Class. Quantum Grav. 15, 2529 (1998).

[15] J.-P. Luminet and B. F. Roukema, Topology of the Universe: Theory and Observation, in NATO ASIC Proc. 541: Theoretical and Observational Cosmology, p. 117, 1999, astro-ph/9901364.

[16] J. Levin, Physics Report 365, 251 (2002). 
[17] M. J. Rebouças and G. I. Gomero, Braz. J. Phys. 34, 1358 (2004), astro-ph/0402324.

[18] J.-P. Luminet, The Shape and Topology of the Universe, in Proceedings of the conference "Tessellations: The world a jigsaw", Leyden (Netherlands), March 2006, 2008, arXiv:0802.2236 [astro-ph].

[19] R. Aurich, S. Lustig, F. Steiner, and H. Then, Class. Quantum Grav. 21, 4901 (2004), arXiv:astro$\mathrm{ph} / 0403597$.

[20] R. Aurich, S. Lustig, F. Steiner, and H. Then, Phys. Rev. Lett. 94, 021301 (2005), arXiv:astro$\mathrm{ph} / 0412407$.

[21] R. Aurich, S. Lustig, and F. Steiner, Class. Quantum Grav. 22, 2061 (2005), arXiv:astro$\mathrm{ph} / 0412569$.

[22] K. M. Górski et al., Astrophys. J. 622, 759 (2005), HEALPix web-site: http://healpix.jpl.nasa.gov/. 